\title{
Surgical treatment of synchronous multiple neuroendocrine lung tumours (case series): is more always better?
}

\author{
Jury Brandolini, Luca Bertolaccini, Alessandro Pardolesi, Piergiorgio Solli \\ Department of Thoracic Surgery, AUSL Romagna Teaching Hospitals, Forlì, Italy \\ Correspondence to: Jury Brandolini, MD. Department of Thoracic Surgery, AUSL Romagna Teaching Hospitals, Via Carlo Forlanini 34, Forlì 47121, \\ Italy. Email: jury.brandolini@gmail.com.
}

\begin{abstract}
Pulmonary neuroendocrine lung tumours are uncommon neoplasm; the presence of multiple and synchronous lesions is rare, and few case report are described in the literature. We present a case series of three patients without particular symptom and radiologic finding of multiple synchronous pulmonary nodules. All the patients underwent surgical treatment; histopathologic examination revealed typical carcinoid tumours in all three cases (one patient has a synchronous atypical carcinoid). No oncological progressions were observed at follow-up. A review of current literature is also presented with a suggested strategy management.
\end{abstract}

Keywords: Synchronous carcinoid; lung cancer; neuroendocrine lung tumours; surgical management

Submitted Jun 26, 2017. Accepted for publication Aug 04, 2017.

doi: $10.21037 /$ atm.2017.08.13

View this article at: http://dx.doi.org/10.21037/atm.2017.08.13

\section{Introduction}

Neuroendocrine tumours (NET) are a rare, heterogeneous group of malignancies that arise from neuroendocrine cells throughout the body, with the lungs and gastrointestinal tract being the most common sites of origin. NET is an uncommon malignant neoplasm, accounting for $1-2 \%$ of all lung tumours. Typically presented as central lesions, a radiological setting of multiple peripheral lesions is uncommon (1). At the moment, surgery is the gold standard of treatment of these tumours, with a different approach between typical carcinoids, in which a parenchyma-sparing resection is preferred, and atypical carcinoids, in which lobectomy should be advocated (2). We described a case series of three patients with synchronous NET of the lung radically treated by surgery.

\section{Case presentation}

\section{Patient \#1}

A 58-year-old female was admitted to our department after the radiological evidence of two lesions of the right hemithorax. The patient was in excellent general condition with no striking finding on physical and laboratory examination. She has no tobacco or alcohol addiction. Chest computed tomography (CT) scan confirmed the two lesions: $18 \mathrm{~mm}$ in diameter in the right upper lobe, and another $14 \mathrm{~mm}$ in diameter in the right lower lobe (Figure 1A,B). A positron emission tomographic (PET) scan did not show uptakes from nodules. Respiratory function tests were normal. Since the lesions were deeply for a minimally invasive approach, a muscle sparing thoracotomy was performed. Two wedge resections with mediastinal lymph nodes dissection were performed; intraoperative frozen section revealed carcinoid in both nodules. Pathological examination confirmed that both nodules were typical carcinoid tumours without lymph nodes metastases. Immunohistochemistry showed that positivity of tumour cells for CD56, synaptophysin, TTF-1, Ki-67 (1\%) and chromogranin (Figure 2). The postoperative course was uneventful, and the patient was discharged on postoperative day 5. Two years follow-up did not reveal any local or distance progression.

\section{Patient \#2}

A 59-year-old female was referred to our hospital with a 

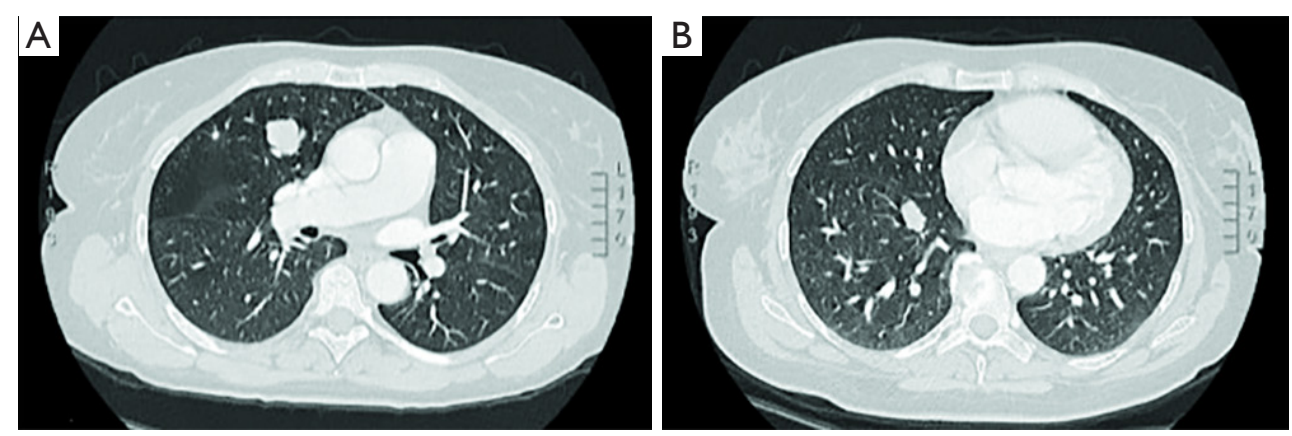

Figure 1 Thorax CT scan (A,B) is showing two nodules of the right upper and lower lobe. CT, computed tomography.

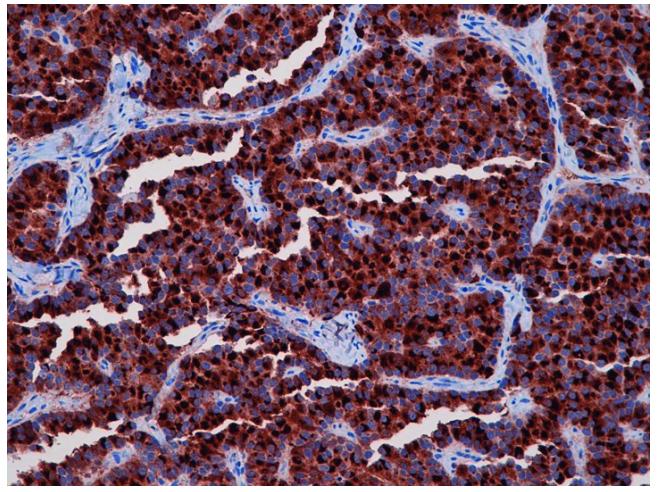

Figure 2 Intense immunoreactivity for chromogranin A showed by tumour cells [OptiView DAB IHC Detection Kit (Ventana, Tucson, USA) on 4-micron section, magnification $\times 20$ ].

nonproductive cough during the previous 2 months. CT scan revealed two nodular lesions in the right lower lobe (20 and $10 \mathrm{~mm}$ in diameter, respectively). An 18-FDG PET showed low metabolic activity [maximum standardized uptake value $($ SUVmax) $=2.9$ ]. Laboratory examination and lung function tests were normal. A fiberoptic bronchoscopy with transbronchial biopsy was performed, with a diagnosis of typical carcinoid tumour. The patient underwent a right lower lobectomy with mediastinal lymphadenectomy by a muscle-sparing thoracotomy. The presence of a $20-\mathrm{mm}$ typical carcinoid was histologically confirmed (synaptophysin, TTF1, chromogranin positive on immunohistochemistry, Ki-67/Mib-1 of 1-2\%), with an unexpected pathologic finding of atypical carcinoid of the smallest nodule and diffuse idiopathic pulmonary neuroendocrine cell hyperplasia (DIPNECH). No lymph node metastasis was detected. After 14 months, the patient is healthy with regular follow-up.

\section{Patient \#3}

A 58-year-old woman was admitted to our department with bilateral lung nodules showed on chest CT scan, seven located in the right lung and four located in the left lung (Figure 3), mediastinal lymphadenopathy was absent. The patient was in good general condition with no smoking habit and no history of the significant systemic disease. Lung metastases of unknown origin were suspected; 18FDG PET revealed reduced uptake only within the two largest nodules of the left upper lobe (SUVmax 6.1) and middle lobe (SUVmax 1.1) (Figure 4). Normal age-related lung function test was showed on spirometry. A preoperative fiberoptic bronchoscopy revealed no pathological findings with negative transbronchial biopsy. After a multidisciplinary tumour board, to obtain a diagnosis, a right lung transthoracic biopsy showed typical carcinoid tumour; therefore, a muscle sparing thoracotomy with a left upper lobectomy with multiple wedge resections in the lower lobe was performed. Postoperative pathological examination confirmed typical carcinoid for all nodules. The patient's postoperative course was regular, and she was discharged after 6 days. A right thoracotomy was performed 3 weeks later after the first operation, and a middle lobectomy with multiple wedge resections in the lower lobe was performed; typical carcinoid tumour was revealed for all seven nodules without lymph node metastases. The patient was discharged on postoperative day 6, and a four years follow-up was uneventful.

\section{Discussion}

NET are rare malignant neoplasm with an approximate annual incidence of 2.3-2.8 cases per million of the population (3). Many carcinoid lesions arise in the lobar 


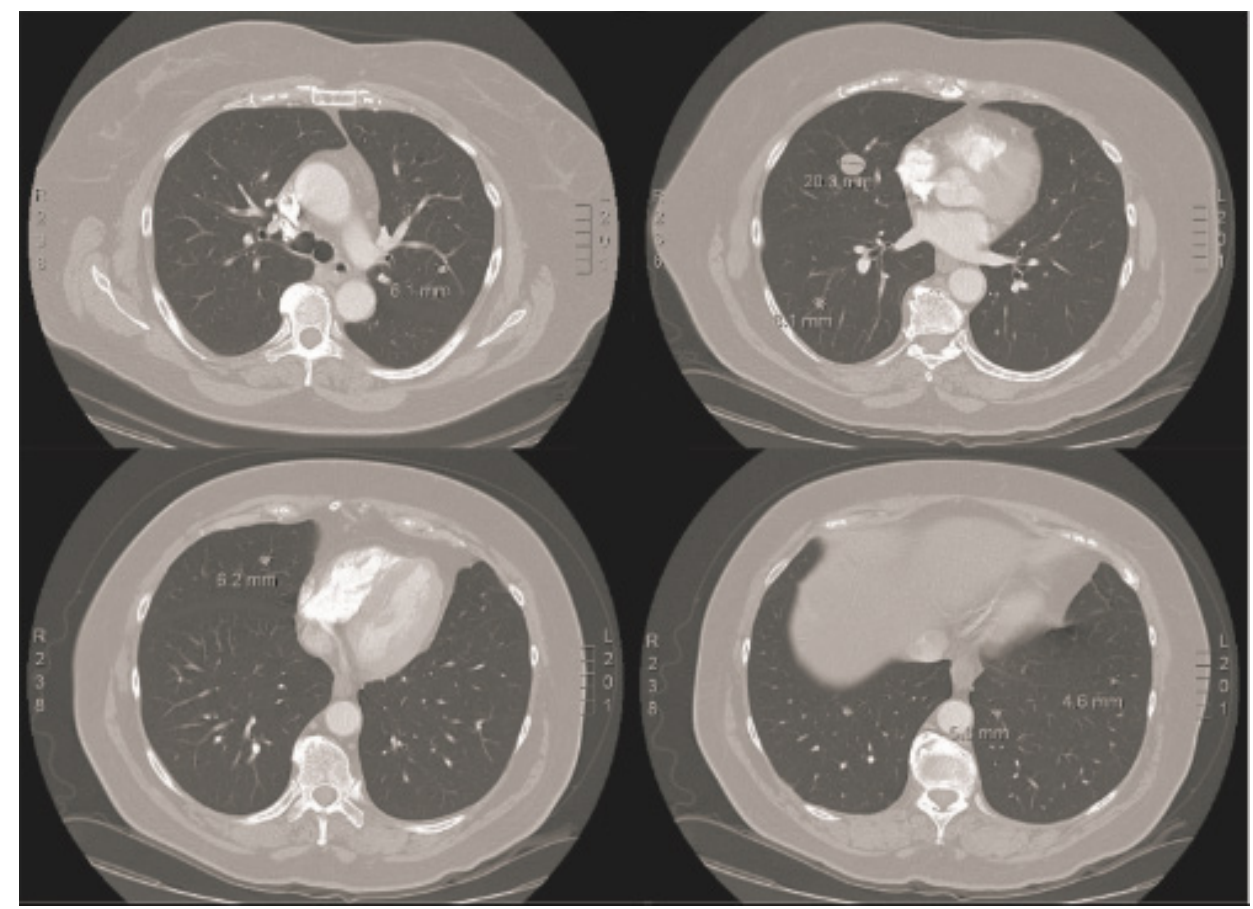

Figure 3 Multiple lung nodules revealed on CT scan.

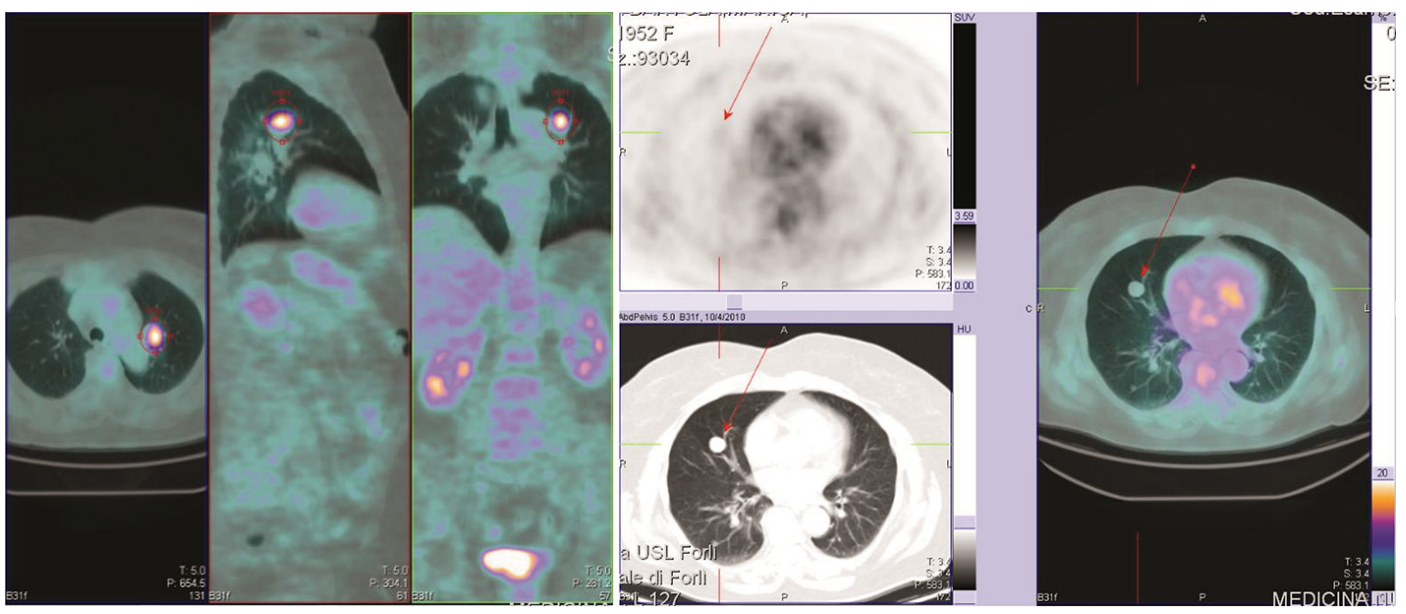

Figure 4 An 18-FDG PET is showing low-grade uptake only within the two largest nodules of LUL (SUVmax 6.1) and ML (SUVmax 1.1). Red arrow, positive enhancement in the ML (SUVmax 1.1). PET, positron emission tomographic; LUL, left upper lobe; ML, middle lobe.

segmental or large segmental bronchus and are typically revealed during endoscopy as polypoid masses (4); typical, synchronous and peripheral carcinoid tumours are sporadic. Due in part to the rarity of lung NET and carcinoids specifically, a relatively limited amount of evidence and accumulated knowledge is available compared with other cancers. Experts from the European Neuroendocrine
Tumor Society, European Society of Thoracic Surgeons, and National Comprehensive Cancer Network agree that a multidisciplinary approach is required for effective and efficient diagnosis and treatment of advanced lung NET. As mentioned previously, the diagnosis and management of lung NET are complicated due to a combination of the asymptomatic nature of lung NET, 
non-specificity of symptoms when present, and variable natural history. It is therefore recommended that each case is assessed in consultation with several experts within a multidisciplinary tumour board specialising in pulmonary carcinoids (including surgeons, medical and radiation oncologists, pulmonologists, pathologists, endocrinologists, interventional radiologists, and gastroenterologists where required) (5).

In case \#3, tumours were widely distributed everywhere in the lung parenchyma suggesting a metastatic disease, which is unusual because carcinoids rarely appear as single solid or nodular lesions in the periphery of the lung. In a review of the literature, we found only six cases with bilateral multiple synchronous carcinoid tumours (1,6-10).

We reported a case in which immunohistochemistry revealed the presence of an atypical carcinoid tumour in a background of DIPNECH within the lung parenchyma. DIPNECH is a rare pulmonary disorder and usually constitutes an unexpected pathologic finding; current best evidence assumed that DIPNECH is probably a preneoplastic condition and that the carcinoid tumours evolved from intraepithelial NECH to carcinoid tumorlets, which grow until $5 \mathrm{~mm}$ in diameter required for classification as carcinoid tumour(s) (11). Close surveillance is the suggested management of patients with DIPNECH; the role of surgical intervention is not delineated (12).

Carcinoid tumours commonly exhibit less aggressive behaviour, with an overall survival of $92 \%$ to $97 \%$ for typical carcinoids and $66 \%$ to $78 \%$ for atypical carcinoids (13). The largest study which includes cases of multiple carcinoid tumours and tumorlets reported by Aubry et al., reveal that they occur most commonly in patients with multiple nodules resembling metastatic disease; long-term survival is excellent, although patients with persistent disease (14). For patients with a carcinoid tumour(s) formation, complete surgical resection of the nodule, in association with systematic mediastinal and hilar node dissection is the best treatment with a good result at the median and long-term survival (9).

To date, several studies have shown that sub-lobar resection with preservation of much of the lung tissue as possible, compared with lobectomy, seem to be justified in the case of multiple, peripheral or bilateral typical carcinoid with at least similar survival and low recurrence rate (15-17). Although metastatic disease mainly causes multiple lung nodules revealed on CT-scan investigation, multiple synchronous carcinoids should be kept in mind. To date, due to its rarity, the exact prognostic impact of a multiplicity of bronchial carcinoids is unknown; about the treatment strategy, we recommend a careful preoperative investigation, complete excision of the tumours with lymph node dissection and adequate long-term follow-up with CT-scan.

As evident by the limited clinical information available regarding lung NET, debate and controversy in classification, diagnostic methods, and appropriate treatment regimens still exist. Along with this research, lung NET guidelines require regular updating (5).

In conclusion, it is important to note that issues surrounding surgical treatment of advanced stage lung NET need to be addressed, along with increasing the awareness among multidisciplinary tumour boards which are on the front line of diagnosis and treatment. Because for a thoracic surgeon, less is not always better; sometimes it is just less.

\section{Acknowledgements}

None.

\section{Footnote}

Conflicts of Interest: The authors have no conflicts of interest to declare.

Informed Consent: Written informed consent was obtained from the patients for publication of this manuscript and any accompanying images.

\section{References}

1. Beshay M, Roth T, Stein R, et al. Synchronous bilateral typical pulmonary carcinoid tumors. Eur J Cardiothorac Surg 2003;23:251-3.

2. Mezzetti M, Raveglia F, Panigalli T, et al. Assessment of outcomes in typical and atypical carcinoids according to latest WHO classification. Ann Thorac Surg 2003;76:1838-42.

3. Cardillo G, Sera F, Di Martino M, et al. Bronchial carcinoid tumors: nodal status and long-term survival after resection. Ann Thorac Surg 2004;77:1781-5.

4. Fraser RS, Pare JA, Fraser RG, et al. Neoplastic disease of the lungs. In: Fraser RS, Pare JA, Fraser RG, et al. editors. Synopsis of Diseases of the Chest. 2nd edition. Philadelphia: WB Saunders, 1994:445-538.

5. Wolin EM. Advances in the Diagnosis and Management of Well-Differentiated and Intermediate-Differentiated 
Neuroendocrine Tumors of the Lung. Chest 2017;151:1141-6.

6. Yazici U, Gulhan E, Agackiran Y, et al. Synchronous bilateral multiple typical pulmonary carcinoid tumors. Ann Thorac Surg 2010;89:1280-8.

7. Sivrikoz MC, Tulay CM, Döner E, et al. Synchronous bilateral typical carcinoid tumors of the lung. Thorac Cardiovasc Surg 2008;56:62-4.

8. Camargo SM, Machuca TN, Moreira AL, et al. Multiple synchronous bronchial carcinoid tumors: report of a case. Thorac Cardiovasc Surg 2009;57:58-60.

9. Akashiba T, Matsumoto K, Kosaka N, et al. Multifocal peripheral bronchial carcinoid tumour. Respirology 1999;4:199-201.

10. Harada H, Yamashita Y, Handa Y, et al. Synchronous, Bilateral, Peripheral, Typical Pulmonary Carcinoid Tumors: Report of a Case and Implications for Management. Int J Med Pharm Case Reports 2014 (2014), Article ID 482878.

11. Wirtschafter E, Walts AE, Liu ST, et al. Diffuse Idiopathic Pulmonary Neuroendocrine Cell Hyperplasia of the Lung (DIPNECH): Current Best Evidence. Lung

Cite this article as: Brandolini J, Bertolaccini L, Pardolesi A, Solli P. Surgical treatment of synchronous multiple neuroendocrine lung tumours (case series): is more always better? Ann Transl Med 2017;5(21):423. doi: 10.21037/ atm.2017.08.13
2015;193:659-67.

12. Irshad S, McLean E, Rankin S, et al. Unilateral diffuse idiopathic pulmonary neuroendocrine cell hyperplasia and multiple carcinoids treated with surgical resection. J Thorac Oncol 2010;5:921-3.

13. Naalsund A, Rostad H, Strøm EH, et al. Carcinoid lung tumors--incidence, treatment and outcomes: a populationbased study. Eur J Cardiothorac Surg 2011;39:565-9.

14. Aubry MC, Thomas CF Jr, Jett JR, et al. Significance of multiple carcinoid tumors and tumorlets in surgical lung specimens: analysis of 28 patients. Chest 2007;131:1635-43.

15. Fox M, Van Berkel V, Bousamra M 2nd, et al. Surgical management of pulmonary carcinoid tumors: sublobar resection versus lobectomy. Am J Surg 2013;205:200-8.

16. Yendamuri S, Gold D, Jayaprakash V, et al. Is sublobar resection sufficient for carcinoid tumors? Ann Thorac Surg 2011;92:1774-8; discussion 1778-9.

17. Ferguson MK, Landreneau RJ, Hazelrigg SR, et al. Longterm outcome after resection for bronchial carcinoid tumors. Eur J Cardiothorac Surg 2000;18:156-61. 